\title{
Carthamin yellow improves cerebral ischemia-reperfusion injury by attenuating inflammation and ferroptosis in rats
}

\author{
HUIHUI GUO ${ }^{1}$, LILI ZHU ${ }^{2}$, PINGPING TANG ${ }^{2},{\text { DONG } \mathrm{CHEN}^{2}, \text { YANCAI }^{2}{ }^{2}, \text { JIANBING }^{2} \text { and CHAO BAO }}^{2}$ \\ ${ }^{1}$ Key Laboratory of Acupuncture and Medicine Research of Ministry of Education, ${ }^{2}$ Department of Acupuncture, \\ Affiliated Hospital of Nanjing University of Chinese Medicine, Nanjing, Jiangsu 210029, P.R. China
}

Received November 14, 2020; Accepted January 20, 2021

DOI: $10.3892 / \mathrm{ijmm} .2021 .4885$

\begin{abstract}
Carthamin yellow (CY), a flavonoid compound extracted from safflower, has been reported to attenuate cardiac ischemia and reperfusion injury. However, whether CY could ameliorate ischemic stroke is not completely understood. In the present study, the preventive effects of $\mathrm{CY}$ on experimental ischemic stroke were investigated using middle cerebral artery occlusion (MCAO) model rats. Neurological scores, brain edema, infarct area and microtubule-associated protein 2 (MAP-2) immunoreactivity were assessed to evaluate the effects of CY on ischemic brain injury. The involvement of inflammation and ferroptosis were examined to investigate the mechanism underlying the effects of CY. The results demonstrated that 2 -week CY treatment attenuated the neurological deficit score, brain water content and infarct area, and increased MAP-2 immunoreactivity in the cortex in MCAO model rats. CY administration also deactivated the cortex NF- $\kappa \mathrm{B} / \mathrm{NLR}$ family pyrin domain containing 3 inflammasome signaling pathway, and decreased serum TNF- $\alpha$, IL-1 $\beta$ and IL- 6 concentrations. Moreover, $\mathrm{CY}$ treatment inhibited $\mathrm{Fe}^{2+}$ and reactive oxygen species accumulation, and reversed acyl-CoA synthetase long-chain family member 4 , transferrin receptor 1 , glutathione peroxidase 4 and ferritin heavy chain 1 protein expression levels in the brain. The levels of glutathione, superoxide dismutase and malondialdehyde in the serum were also reversed by CY treatment. Collectively, the results of the present study demonstrated that CY protected rats against ischemic stroke, which was associated with mitigation of inflammation and ferroptosis.
\end{abstract}

Correspondence to: Professor Chao Bao or Professor Jianbing Li, Department of Acupuncture, Affiliated Hospital of Nanjing University of Chinese Medicine, 155 Hanzhong Road, Nanjing, Jiangsu 210029, P.R. China

E-mail: drbaochao@163.com

E-mail: njdrli@163.com

Key words: ischemic stroke, carthamin yellow, microtubule-associated protein 2, ferroptosis, NF- $\mathrm{kB} / \mathrm{NLRP} 3$ inflammasome

\section{Introduction}

Ischemic stroke, which accounts for $85 \%$ of all stroke cases, is the leading cause of destructive cerebrovascular disease, and displays high mortality and morbidity rates (1). To date, pharmacological thrombolysis is the most effective treatment, but displays a golden treatment time, meaning only 3-8\% of patients are eligible for this therapy. Therefore, novel therapies against ischemic stroke are required (2). Previous studies have indicated that inflammation and ferroptosis serve significant roles in the development of ischemic stroke $(3,4)$.

The NLR family pyrin domain containing 3 (NLRP3) inflammasome, which consists of NLRP3, apoptosis-associated speck-like protein (ASC) and caspase-1, is an important component of the innate immune system (5). The NLRP3 inflammasome triggers the production of the proinflammatory cytokine, interleukin (IL)-1 $\beta$, which facilitates the secretion of inflammatory mediators, including tumor necrosis factor (TNF) $\alpha$ and IL-6 (6). NF- $\kappa \mathrm{B}$ is a transcription factor that alters the formation of the NLRP3 inflammasome, and the $\mathrm{NF}-\kappa \mathrm{B} / \mathrm{NLRP} 3$ inflammasome signaling is a key mechanism of ischemic stroke (7). Hippocampal NF- $\kappa \mathrm{B}$ mRNA expression, and serum TNF- $\alpha$ and IL- $1 \beta$ levels were increased in response to ischemia stroke (8). Cerebral ischemia-reperfusion injury activated the NLRP3 inflammasome proteins in rats, which could be suppressed by electroacupuncture, leading to improvement of neurological deficit scores and infarct sizes in middle cerebral artery occlusion (MCAO) model rats (9).

Ferroptosis is a novel type of cell death driven by iron-dependent accumulation of lipid-based reactive oxygen species (ROS), which is regulated by the inactivation of glutathione peroxidase 4 (GPX4) that can reduce lipid peroxides at the expense of glutathione (GSH) (10). Numerous proteins are involved in ferroptosis, including acyl-CoA synthetase long-chain family member 4 (ACSL4), transferrin receptor 1 (TFR1) and ferritin heavy chain 1 (FTH1) $(11,12)$. ACSL4 is a lipid metabolism enzyme that is required for ferroptosis, contributing to lipid peroxidation and ferroptosis (13). TFR1 transports iron from the extracellular environment into cells, facilitating the cellular iron pool essential for ferroptosis (14). FTH1 is a major iron storage protein responsible for maintaining the iron balance in cells (15). Ferroptosis is implicated in ischemic stroke $(4,16)$. Increased ROS and iron levels were observed in the brain of ischemic stroke model rodents (17). 
Carthamin yellow (CY) is a flavonoid compound isolated from safflower. In Traditional Chinese medicine, it is considered that CY improves blood circulation and alleviates pain; thus, CY is used for the treatment of coronary heart disease, cerebrovascular disease and angiitis in China (18). Antioxidant and anti-inflammatory properties of CY have been reported. CY inhibited lipopolysaccharide-induced activation of TNF- $\alpha$ (19). In vivo and in vitro studies demonstrated that $\mathrm{CY}$ protected against cardiac ischemia and reperfusion injury via decreasing ROS release and NLRP3 inflammasome-related inflammatory responses (18). The aforementioned studies indicated that whether CY could ameliorate ischemic stroke by attenuating inflammation and ferroptosis required further investigation. Therefore, the present study investigated the protective effect of $\mathrm{CY}$ in ischemic stroke by using MCAO model rats, and explored the involvement of inflammation and ferroptosis in CY-mediated effects to explore the possible underlying mechanisms.

\section{Materials and methods}

Animals. A total of 32 male Sprague-Dawley rats (aged 6-8 weeks; 250-280 g) were purchased from Shanghai Sipper-BK Lab Animal Co., Ltd. Animals were housed in an animal center at a controlled temperature $\left(23 \pm 1^{\circ} \mathrm{C}\right)$ and humidity $(60 \pm 2 \%)$ with 12-h light/dark cycles, and free access to food and water. All experiments were conducted in compliance with the Provision and General Recommendation of Chinese Experimental Animals Administration Legislation and approved by the Institutional Animal Care and Use Ethics Committee at Nanjing University of Chinese Medicine (approval no. 201907A544).

Materials. CY was obtained from Sigma-Aldrich (Merck KGaA; Fig. 1). All antibodies were purchased from Cell Signaling Technology, Inc., ProteinTech Group, ABclonal Technology, Inc. or Abcam.

Experimental protocol. Animals were randomly divided into the following four groups ( $\mathrm{n}=8$ per group): i) Sham; ii) MCAO; iii) CY (20 mg/kg); and iv) CY (40 mg/kg). CY was administered intragastrically to rats once daily for 2 weeks. At $60 \mathrm{~min}$ after the last administration, MCAO surgery was performed as previously described (20). At 24 h post-reperfusion, neurological scores, brain water content and infarct volume were determined. Immunofluorescence staining, western blotting and flow cytometry were performed to investigate the potential mechanisms. After neurological scoring, rats were euthanized with $400 \mathrm{mg} / \mathrm{kg}$ pentobarbital sodium. The brains were immediately removed, and the cortex and the serum were collected and stored at $-80^{\circ} \mathrm{C}$ until further use.

Neurological scoring. For the evaluation of functional recovery, neurological scores were assessed at $24 \mathrm{~h}$ post-MCAO induction by an observer blinded to the treatments as previously reported (20). The neurological scoring ranged from 0 to 4 (normal score, 0; maximal deficit score, 4).

Infarct area assessment. Rats were sacrificed and the brains were rapidly removed. Coronal sections were cut into 2-mm thick slices and stained with 1\% 2,3,5-triphenyltetrazolium chloride (TTC; Sigma-Aldrich; Merck KGaA) for $30 \mathrm{~min}$ at $37^{\circ} \mathrm{C}$ followed by fixation with $10 \%$ paraformaldehyde for $10 \mathrm{~min}$. Infarction size was assessed using ImageJ software (version 1.52; National Institutes of Health). The size of infarct regions was calculated using the following equation: Infarct rate $(\%)=$ Infarct volume/total volume $\mathrm{x} 100$.

Brain water content determination. The wet-dry method was applied to determine brain edema. The brains were immediately removed and weighed to obtain the wet weight. Following drying in an oven at $100^{\circ} \mathrm{C}$ for $>24 \mathrm{~h}$, the brains were weighed to obtain the dry weight. The percentage of brain water content was calculated using the following formula: Brain water content $(\%)=[($ wet weight $)-($ dry weight $)] /($ wet weight $) \times 100$.

Immunofluorescence staining. Immunofluorescence staining was performed to detect microtubule-associated protein 2 (MAP-2) and NF- $\mathrm{B}$ expression as previously described (21). Briefly, brain tissues were obtained, fixed with $4 \%$ paraformaldehyde for $24 \mathrm{~h}$ at room temperature and transferred to $30 \%$ sucrose solution. Subsequently, the tissues were frozen in a cryostat machine and cut into frozen sections (10- $\mu \mathrm{m}$ thick) at $-20^{\circ} \mathrm{C}$. Slices were washed with $0.01 \mathrm{M}$ PBS for $5 \mathrm{~min}$, blocked with 5\% goat serum (cat. no. SL038; Solarbio Life Sciences, Inc.) for $1 \mathrm{~h}$ at room temperature, and then incubated with rabbit anti-mouse primary antibodies targeted against MAP-2 (1:50; product no. 4542) and phosphorylated (p)-NF-кB p65 (1:500; product no. 3039; both from Cell Signaling Technology, Inc.) at $4^{\circ} \mathrm{C}$ overnight. After washing, samples were incubated with a goat anti-rabbit Alexa-Fluor IgG secondary antibody (1:100; cat. no. SA00003-2; ProteinTech Group, Inc.) for $1 \mathrm{~h}$ at room temperature. Nuclei were stained with DAPI for $10 \mathrm{~min}$ at room temperature. Images of the injured cortex were observed under high-power fields (magnification, x200; Olympus BX53). The percentage of MAP-2- or $\mathrm{p}-\mathrm{NF}-\kappa \mathrm{B}$ p65-positive staining was determined and analyzed using ImageJ software (version 1.52; National Institutes of Health).

Western blotting. Western blotting was performed as previously described (22). Total protein was isolated from samples using TRIzol $^{\circledR}$ (Invitrogen; Thermo Fisher Scientific, Inc.). Nuclear proteins were extracted using Nuclear and Cytoplasmic Protein Extraction reagents (Thermo Fisher Scientific, Inc.) according to the manufacturer's protocol. Protein concentrations were measured using the BCA Protein Assay kit (Thermo Fisher Scientific, Inc.). Equal amounts of protein $(50 \mu \mathrm{g})$ were separated via $10 \%$ or $12 \%$ SDS-PAGE and transferred to PVDF membranes. After blocking with 5\% BSA (cat. no. A8020; Solarbio Life Sciences, Inc.) for $1 \mathrm{~h}$ at room temperature, the membranes were incubated at $4^{\circ} \mathrm{C}$ overnight with antibodies targeted against:

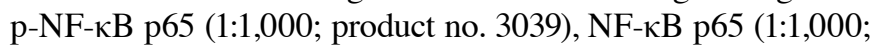
product no. 8242), p-IкB $\alpha$ (1:1,000; product no. 5209), ІкB $\alpha$ (1:1,000; cat. no. 4812; all from Cell Signaling Technology, Inc.), NLRP3 (1:1,000; product code ab263899; Abcam), caspase-1 (1:1,000; cat. no. 22915-1-AP; ProteinTech Group, Inc.), IL-1 $\beta$ (1:1,000; cat. no. A1112; ABclonal Technology, Inc.), ACSL4 (1:1,000; cat. no. A14439; ABclonal Technology, Inc.), FTH1 (1:1,000; product no. 4393; Cell Signaling Technology, Inc.), GPX4 (1:1,000; cat. no. A1933; ABclonal Technology, Inc.), TFR1 (TFRC; 1:1,000; cat. no. A5865; ABclonal Technology, Inc.), $\beta$-tubulin (1:5,000; cat. no. 10068-1-AP; ProteinTech 


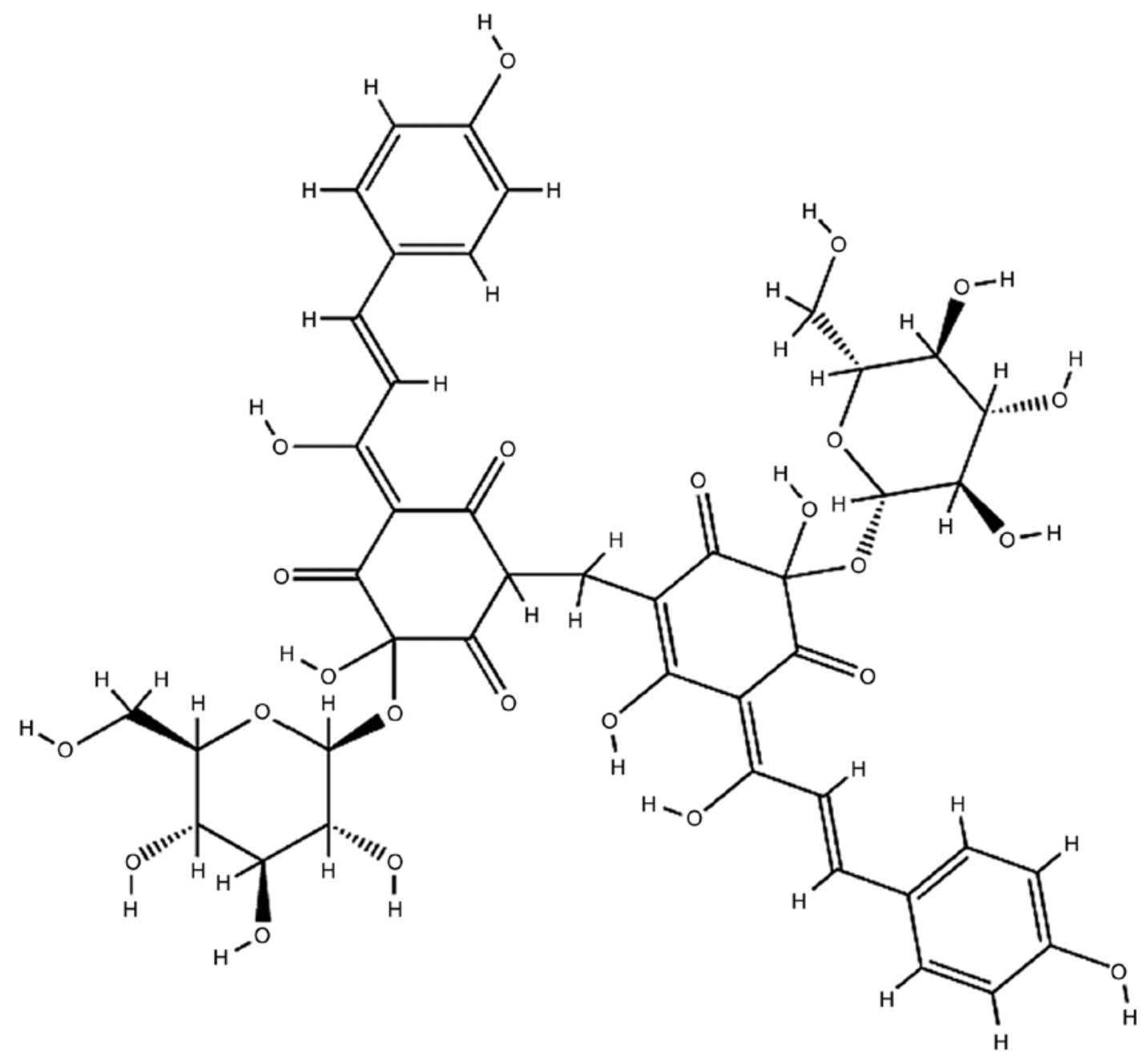

Figure 1. Chemical structure of carthamin yellow.

Group, Inc.) and Lamin A (1:1,000; cat. no. ab8980; Abcam). After washing with TBST (0.1\% Tween-20), the membranes were incubated with appropriate secondary antibody (1:5,000; cat. no. SA00001-2; ProteinTech Group, Inc.) for $1 \mathrm{~h}$ at room temperature. Protein bands were visualized using an ECL kit (cat. no. 180-501; Tanon Science \& Technology Co., Ltd.). Protein expression levels were semi-quantified using ImageJ software (version 1.52; National Institutes of Health) with $\beta$-tubulin or Lamin A as the loading control.

Determination of TNF- $\alpha, I L-1 \beta, I L-6$, malondialdehyde (MDA), GSH and superoxide dismutase (SOD) concentrations in serum. The levels of TNF- $\alpha$ (TNF- $\alpha$ ELISA Kit; cat. no. PT516), IL-1 $\beta$ (IL-1 $\beta$ ELISA Kit; cat. no. PI303), IL-6 (IL-6 ELISA Kit; cat. no. PI328), MDA (MDA detection kit; cat. no. S0131), GSH (GSH detection kit; cat. no. S0052) and SOD (SOD detection kit; cat. no. S0101) in serum were detected using different detection kits (Beyotime Institute of Biotechnology) according to the manufacturer's protocol.

ROS measurement. ROS content was assayed using 2,7-dichlorofluorescin diacetate (DCF), a ROS detection kit (cat. no. H131224; Shanghai Aladdin Bio-Chem Technology Co., Ltd.) according to the manufacturer's protocol.
Iron assay. Iron concentration was assessed using an Iron Assay kit (cat. no. MAK025-1KT; Sigma-Aldrich; Merck $\mathrm{KGaA}$ ) according to the manufacturer's instructions.

Statistical analysis. Statistical analysis was performed using GraphPad Prism software (version 6; GraphPad Software, Inc.). Data are presented as the mean \pm SEM. Comparisons among multiple groups were analyzed using one-way ANOVA followed by Bonferroni's post hoc test. $\mathrm{P}<0.05$ was considered to indicate a statistically significant difference.

\section{Results}

CY improves neurological scores. To determine whether CY induced neurological function recovery in MCAO model rats, neurological scores were evaluated at $24 \mathrm{~h}$ post-reperfusion (Fig. 2A). MCAO model rats displayed increased neurological deficit scores compared with the Sham group, whereas MCAO model-induced neurological deficits were significantly relieved following treatment with $\mathrm{CY}$. The results suggested that $\mathrm{CY}$ improved neurological performance in MCAO model rats.

CY decreases infarction volume and brain water content. $\mathrm{MCAO}$ induced a noticeable increase in brain water content 

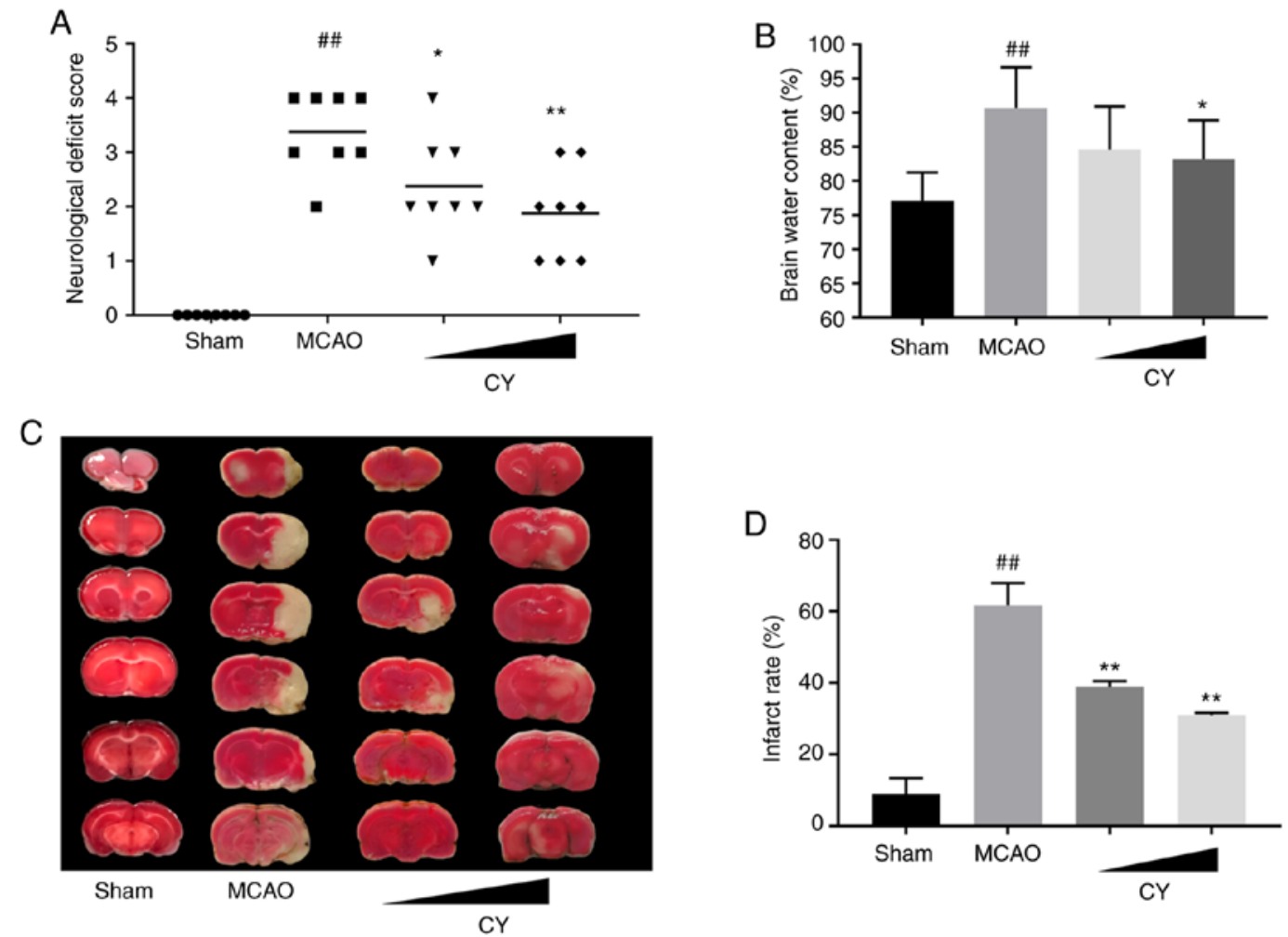

Figure 2. Effects of $\mathrm{CY}$ on neurological deficit scores, brain water content and infarct area. (A) Neurological deficit score. (B) Brain water content. (C and D) Infarct area. Data are presented as the mean \pm SEM. ${ }^{\# \#} \mathrm{P}<0.01$ vs. Sham; ${ }^{*} \mathrm{P}<0.05$ and ${ }^{* *} \mathrm{P}<0.01$ vs. MCAO. CY, carthamin yellow; MCAO, middle cerebral artery occlusion.

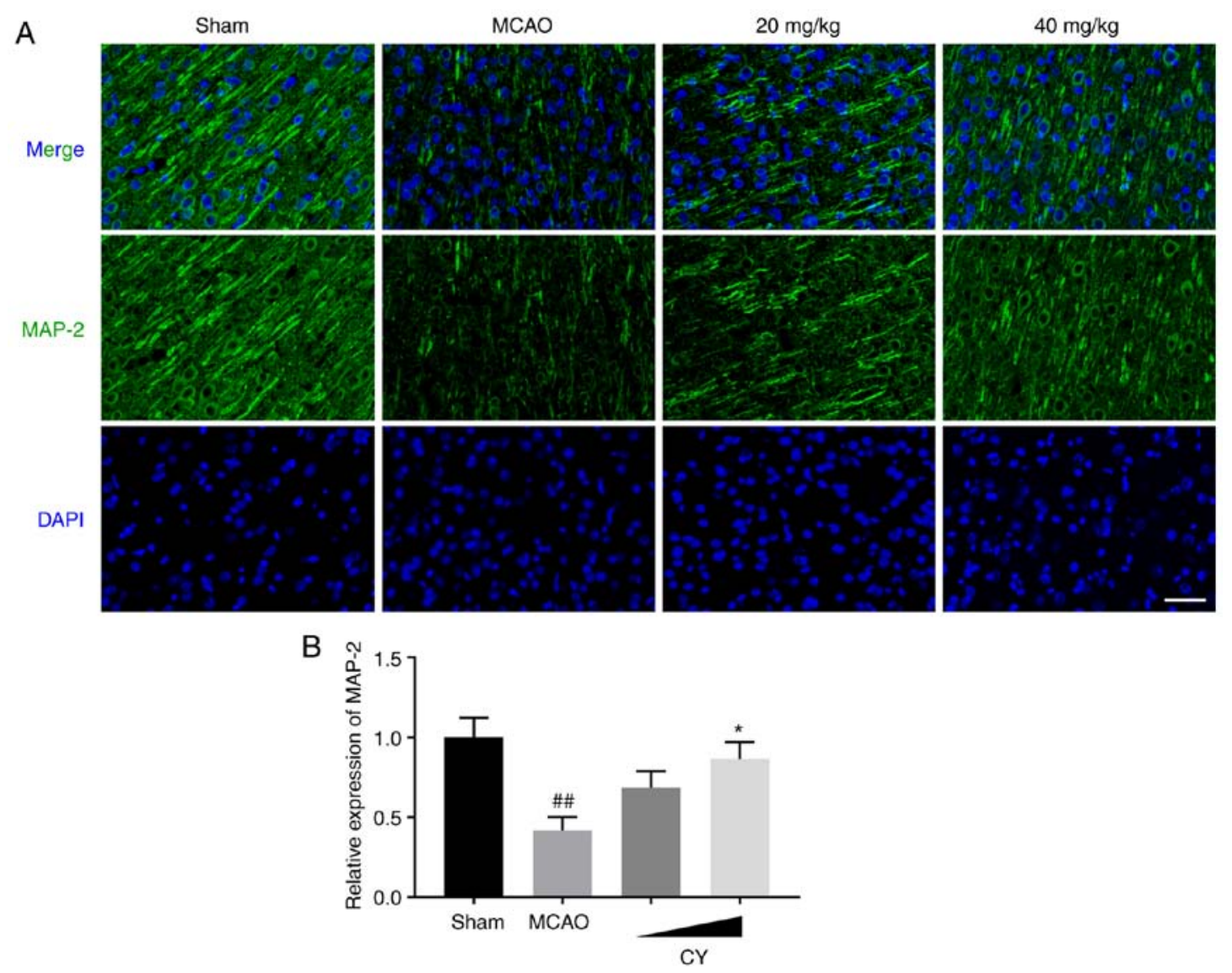

Figure 3. MAP-2 expression in the cortex of MCAO model rats. (A) Representative images of immunofluorescence staining for MAP-2. Scale bar, $50 \mu \mathrm{m}$. (B) Quantification of MAP-2. Data are presented as the mean \pm SEM. ${ }^{* \#} \mathrm{P}<0.01$ vs. Sham; ${ }^{*} \mathrm{P}<0.05$ vs. MCAO. MAP-2, microtubule-associated protein 2; $\mathrm{MCAO}$, middle cerebral artery occlusion; $\mathrm{CY}$, carthamin yellow. 

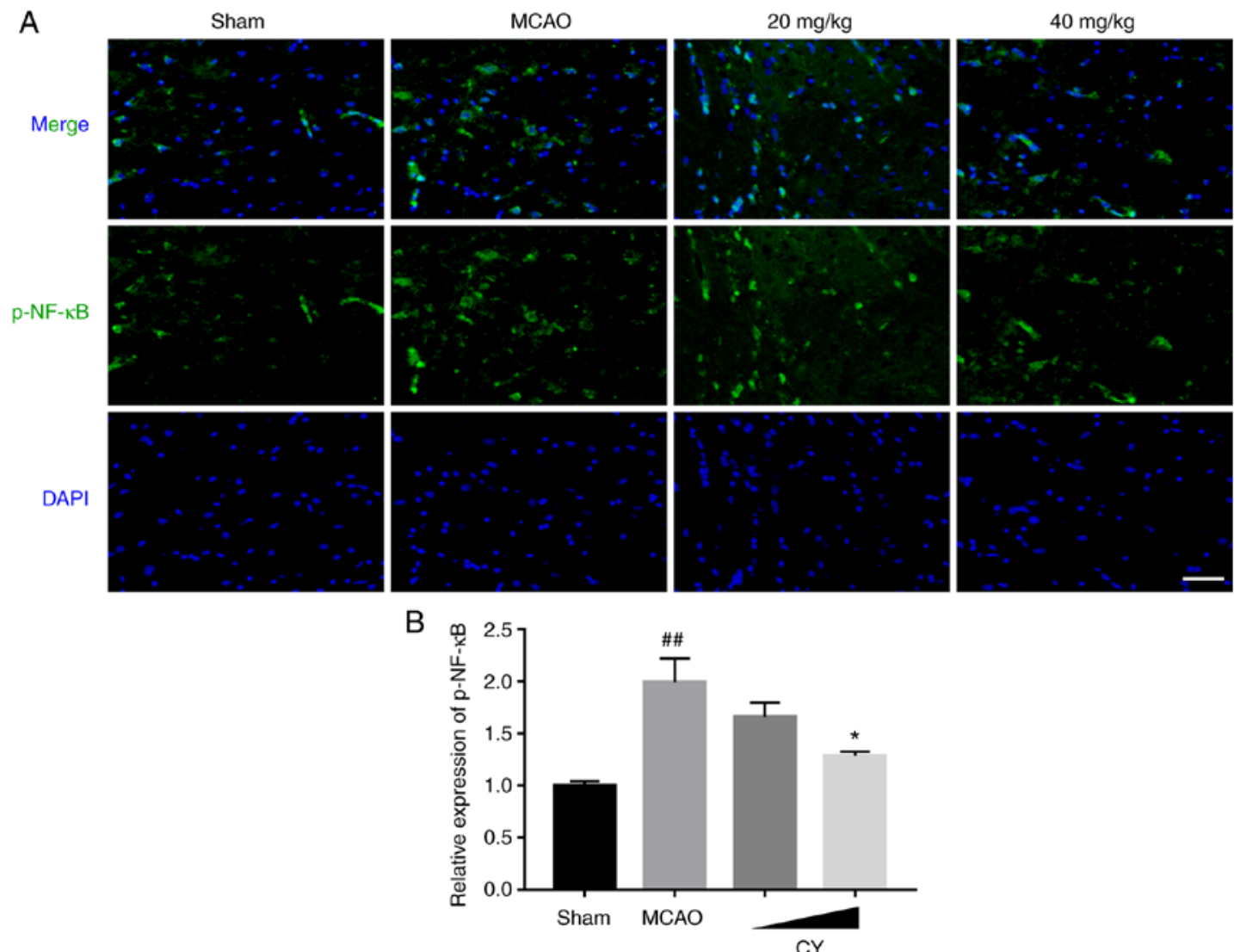

CY

Figure 4. p-NF- $\kappa \mathrm{B}$ expression in the cortex of MCAO model rats. (A) Representative images of immunofluorescence staining for $\mathrm{p}-\mathrm{NF}-\kappa \mathrm{B}$. Scale bar, $50 \mu \mathrm{m}$. (B) Quantification of p-NF-кB. Data are presented as the mean \pm SEM. ${ }^{\# \#} \mathrm{P}<0.01$ vs. Sham; ${ }^{*} \mathrm{P}<0.05$ vs. MCAO. p, phosphorylated; MCAO, middle cerebral artery occlusion; CY, carthamin yellow.

in the ischemic hemisphere at $24 \mathrm{~h}$ post-reperfusion, which was reduced following CY treatment (Fig. 2B). The infarct volume at $24 \mathrm{~h}$ post-reperfusion was determined by performing TTC staining. The results revealed that the infarct area percentage of the brain tissue in MCAO model rats was significantly higher compared with the Sham group, and lower in the CY administration groups compared with MCAO model rats (Fig. 2C and D). The results indicated that CY effectively attenuated the cerebral infarction area and brain water content in MCAO model rats.

$C Y$ increases MAP-2 expression in the cortex. To examine the protective effects of $\mathrm{CY}$ on neurons, immunofluorescence staining for MAP2 was performed. Obvious MAP-2 immunostaining was observed in the cortex of Sham rats (Fig. 3). However, MAP-2 expression in MCAO model rats at $24 \mathrm{~h}$ post-reperfusion was decreased compared with the Sham group. Interestingly, CY successfully enhanced MAP-2 expression following administration for 14 days. The results suggested that $\mathrm{CY}$ prevented neuronal damage in MCAO model rats.

$C Y$ inhibits inflammation in the cortex. Subsequently, immunofluorescence staining, western blotting and ELISAs were performed to investigate the effects of CY on inflammation. The results demonstrated that in the cortex of MCAO model rats, $\mathrm{p}-\mathrm{NF}-\kappa \mathrm{B}$ p65 expression detected via immunofluorescence staining, as well as the protein expression levels of
p-NF- $\kappa$ B p65, p-IкB $\alpha$, NLRP3, caspase-1 and IL-1 $\beta$ detected via western blotting were significantly increased compared with the Sham group (Figs. 4 and 5). Moreover, NF- $\kappa$ B p65 expression in the nucleus was significantly increased compared with the Sham group. Nonetheless, CY administration successfully reversed MCAO model-induced effects. In addition, the MCAO group displayed evidently higher serum levels of TNF- $\alpha$, IL-1 $\beta$ and IL-6 (Fig. 6). Notably, following CY treatment, a marked decrease in TNF- $\alpha$, IL-1 $\beta$ and IL-6 serum levels was observed. Collectively, the results demonstrated that $\mathrm{CY}$ treatment inhibited the inflammatory response in the cortex of MCAO model rats.

CY attenuates ferroptosis. To determine the involvement of ferroptosis in MCAO model rats, iron and ROS accumulation, lipid peroxidation levels and the expression levels of ferroptosis-related proteins were assessed. MCAO model rats displayed obvious ROS and iron accumulation in the cortex compared with Sham rats (Fig. 7). In addition, increased MDA generation, but reduced GSH levels and SOD activities in the serum were observed in MCAO model rats (Fig. 8). The western blotting results indicated that MCAO challenge significantly increased the protein expression levels of ACSL4 and TFR1 in the cortex, but decreased the protein expression levels of FTH1 and GPX4 (Fig. 9). The aforementioned effects induced by MCAO modeling were significantly ameliorated by treatment with $\mathrm{CY}$, indicating the antiferroptosis efficacy of CY in MCAO model rats. 

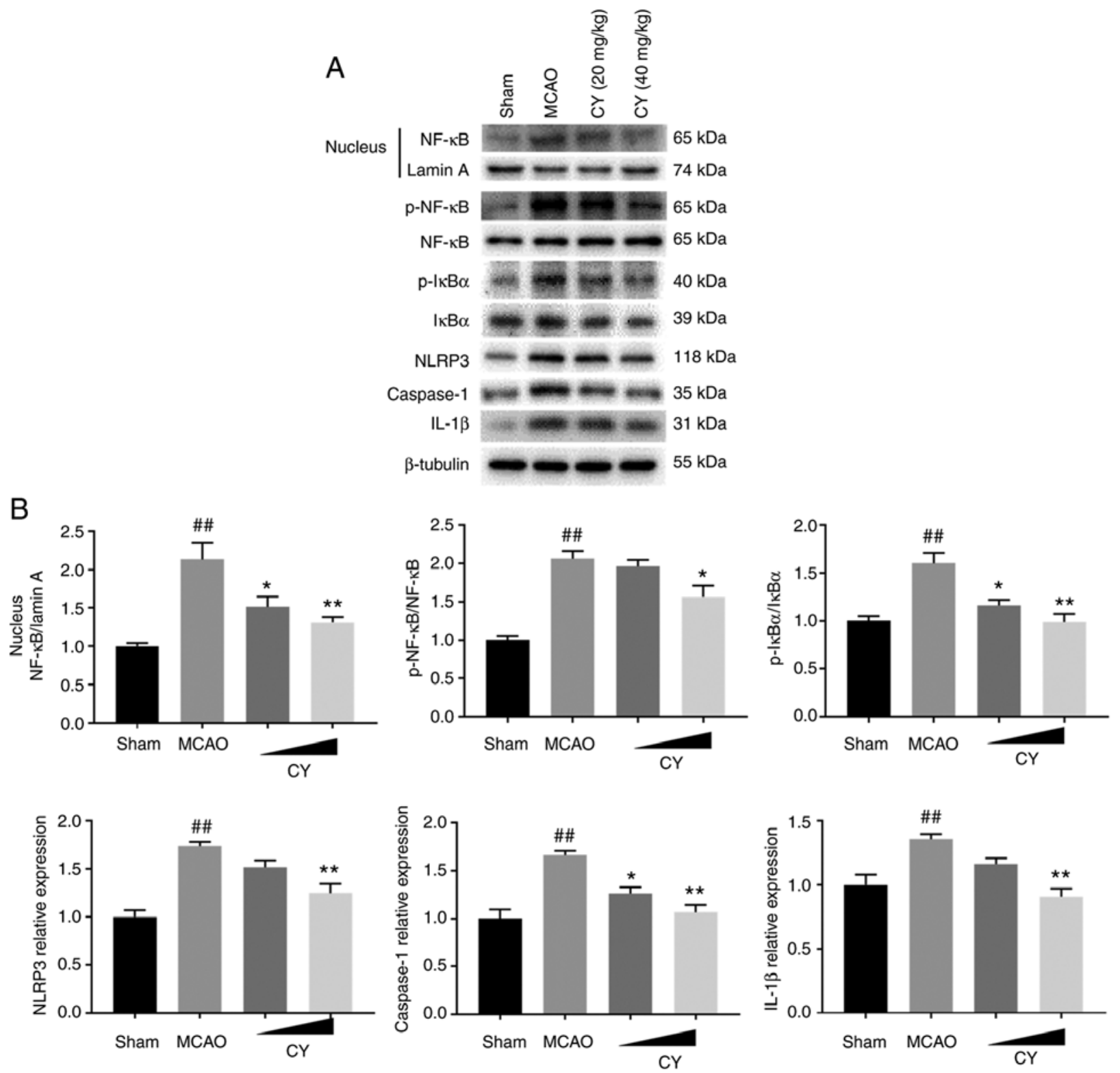

Figure 5. Effects of CY on the NF- $\mathrm{BB} / \mathrm{NLRP} 3$ inflammasome signaling pathway in the cortex. NF- $\mathrm{kB} / \mathrm{NLRP} 3$ inflammasome signaling pathway-related protein expression levels were (A) determined via western blotting and (B) semi-quantified. Data are presented as the mean \pm SEM. ${ }^{\# \#} \mathrm{P}<0.01 \mathrm{vs}$. Sham; $\mathrm{P}<0.05$ and ${ }^{* *} \mathrm{P}<0.01$ vs. MCAO. CY, carthamin yellow; NLRP3, NLR family pyrin domain containing 3; MCAO, middle cerebral artery occlusion; p, phosphorylated.
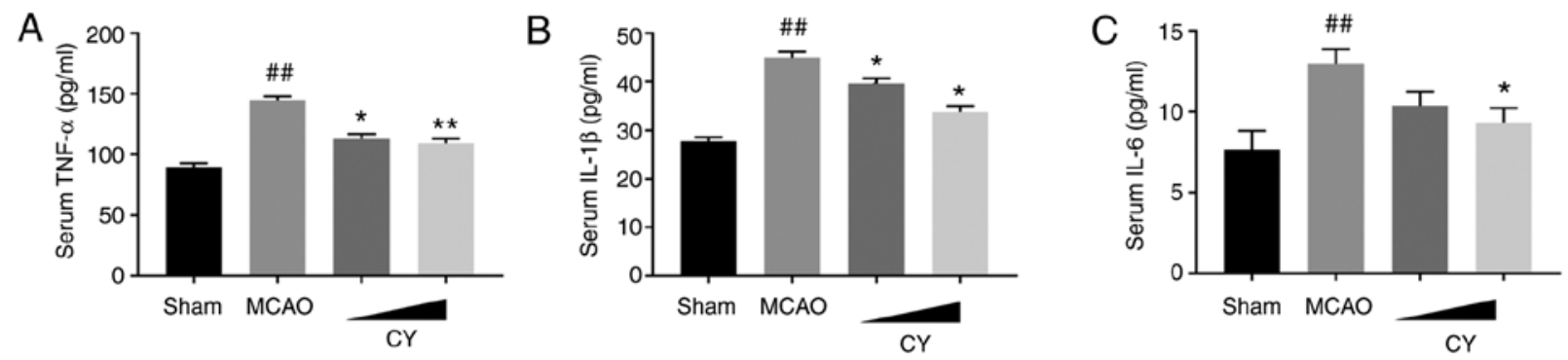

Figure 6. TNF- $\alpha$, IL-1 $\beta$ and IL-6 concentrations in serum. (A) TNF- $\alpha$, (B) IL-1 $\beta$ and (C) IL-6. Data are presented as the mean \pm SEM. ${ }^{\# \#}$ P $<0.01$ vs. Sham; ${ }^{*} \mathrm{P}<0.05$ and ${ }^{* *} \mathrm{P}<0.01$ vs. MCAO. TNF, tumor-necrosis factor; IL, interleukin; MCAO, middle cerebral artery occlusion; CY, carthamin yellow.

\section{Discussion}

In the present study, CY-mediated effects on experimental ischemic stroke were investigated. CY improved neurological deficit scores, brain water content, infarct area and MAP-2 expression in MCAO model rats. $\mathrm{CY}$ also deactivated the NF- $\mathrm{BB} / \mathrm{NLRP} 3$ inflammasome signaling pathway in the cortex. In addition, CY treatment decreased the serum 

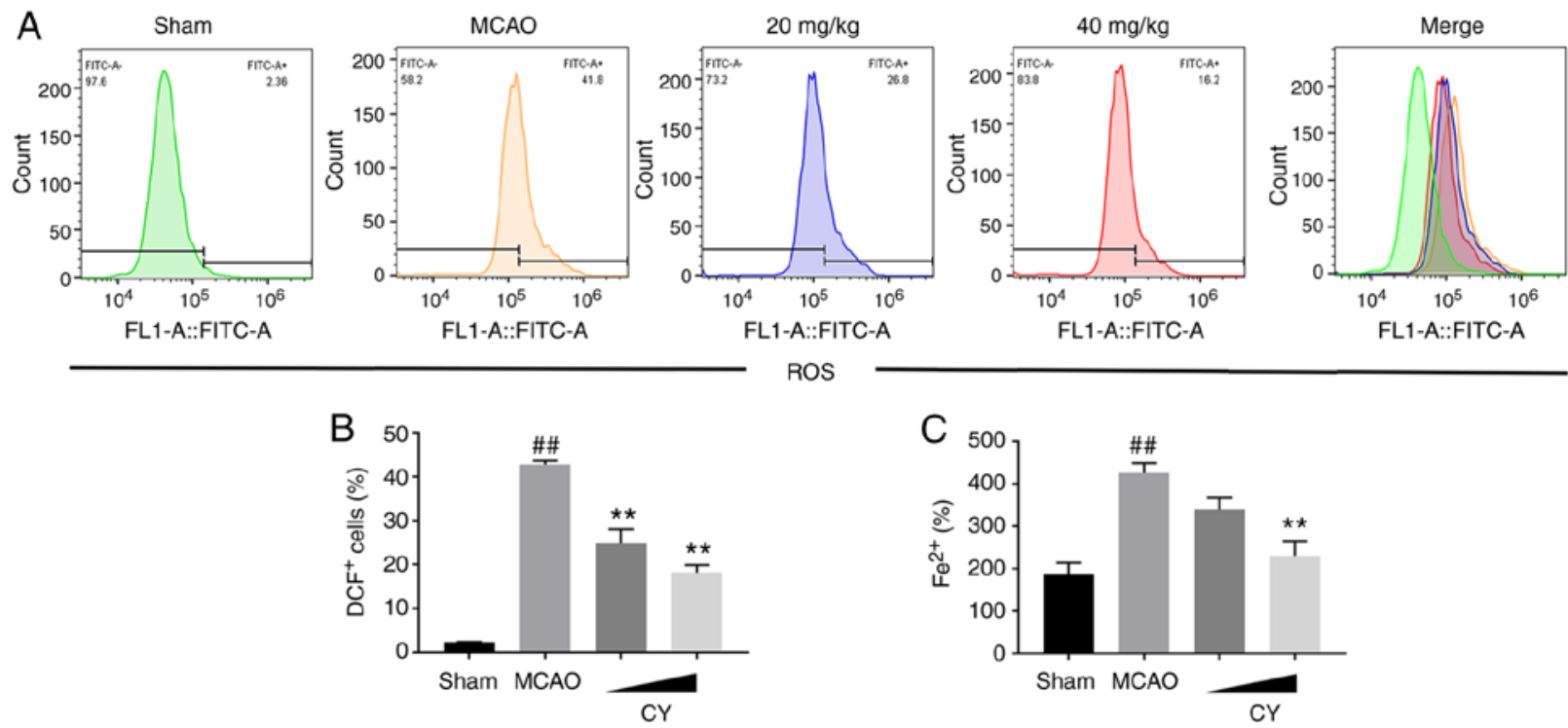

Figure 7. ROS and iron accumulation in the cortex. (A and B) ROS generation. (C) $\mathrm{Fe}^{2+}$ production. Data are presented as the mean $\pm \mathrm{SEM}$. ${ }^{\# \prime} \mathrm{P}<0.01 \mathrm{vs}$. Sham; ${ }^{* *} \mathrm{P}<0.01$ vs. MCAO. ROS, reactive oxygen species; MCAO, middle cerebral artery occlusion; $\mathrm{CY}$, carthamin yellow; DCF, 2,7-dichlorofluorescin diacetate.
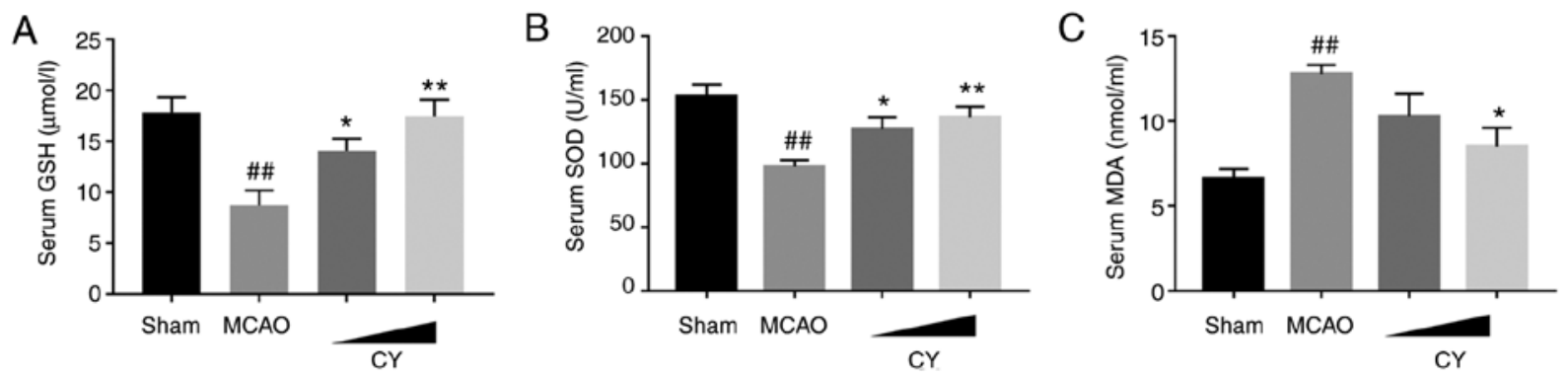

Figure 8. Lipid peroxidation level in serum. (A) GSH, (B) SOD and (C) MDA. Data are presented as the mean \pm SEM. ${ }^{\# \#} \mathrm{P}<0.01$ vs. Sham; ${ }^{*} \mathrm{P}<0.05$ and ${ }^{* * *} \mathrm{P}<0.01$ vs. MCAO. GSH, glutathione; SOD, superoxide dismutase; MDA, malondialdehyde; MCAO, middle cerebral artery occlusion; $\mathrm{CY}$, carthamin yellow.
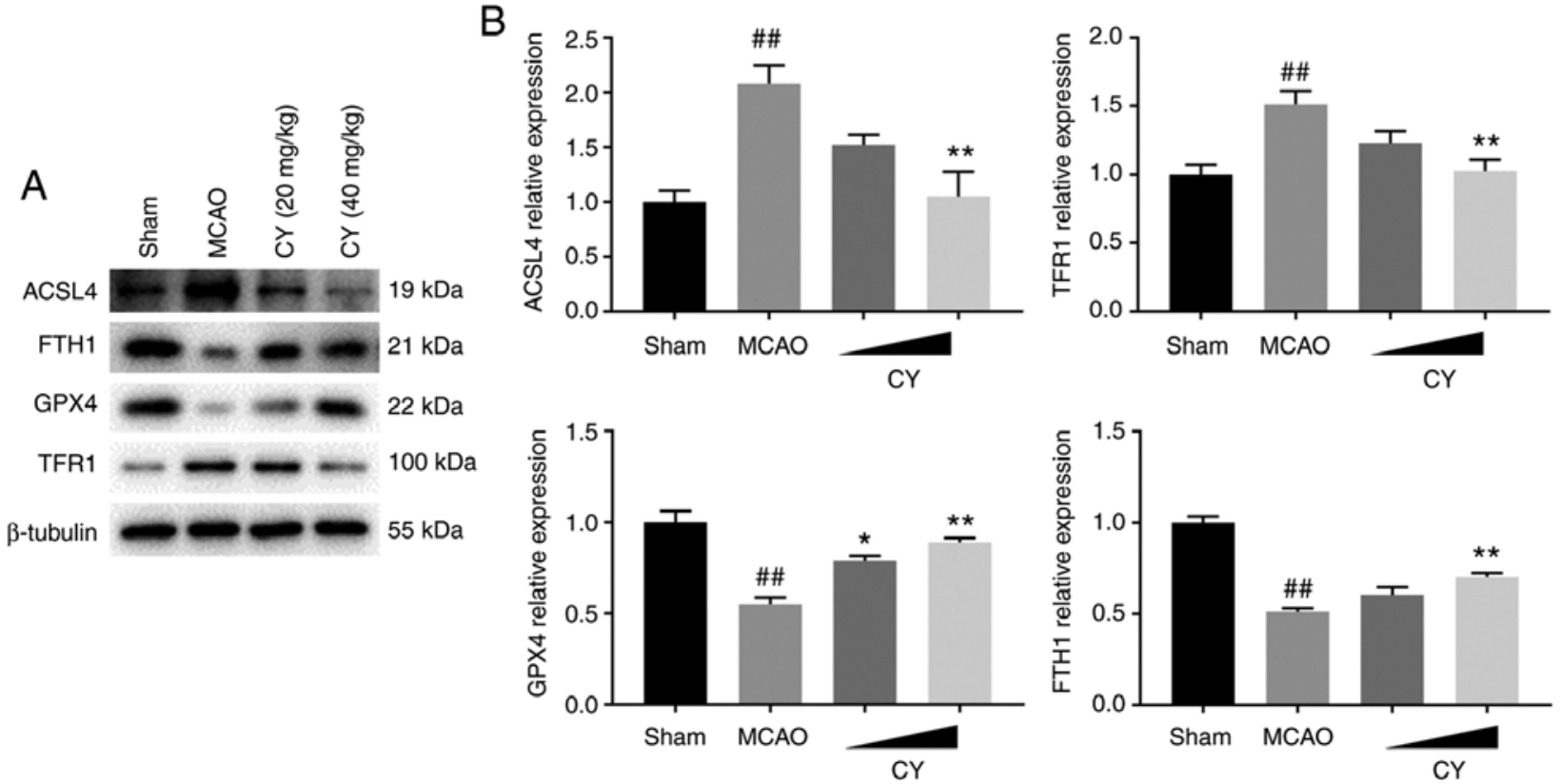

Figure 9. Expression levels of ferroptosis-related proteins in the cortex. Ferroptosis-related protein expression levels were (A) determined via western blotting and (B) semi-quantified. Data are presented as the mean \pm SEM. ${ }^{\# \#} \mathrm{P}<0.01$ vs. Sham; ${ }^{*} \mathrm{P}<0.05$ and ${ }^{* *} \mathrm{P}<0.01$ vs. MCAO. MCAO, middle cerebral artery occlusion; CY, carthamin yellow; ACSL4, acyl-CoA synthetase long-chain family member 4; FTH1, ferritin heavy chain 1; GPX4, glutathione peroxidase 4; TFR1, transferrin receptor. 


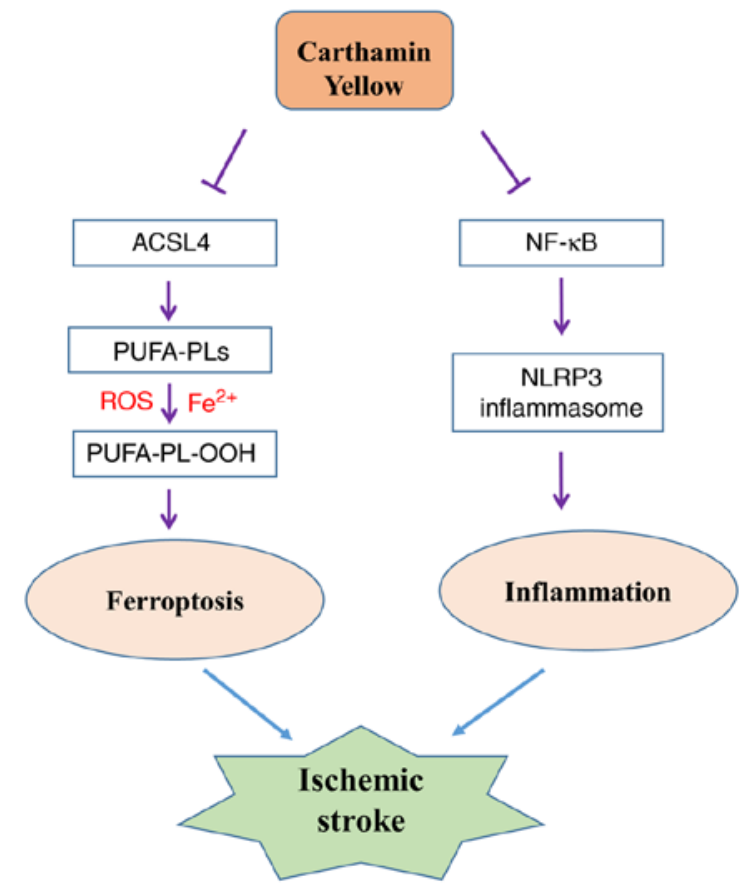

Figure 10. Schematic illustration of the proposed mechanism underlying CY-mediated protection against cerebral ischemia-reperfusion injury. CY, carthamin yellow; ACSL4, acyl-CoA synthetase long-chain family member 4; NLRP3, NLR family pyrin domain containing 3; PUFA, polyunsaturated fatty acids; PL, phospholipid.

concentrations of TNF- $\alpha$, IL-1 $\beta$ and IL-6. CY also alleviated MCAO-induced ferroptosis, as demonstrated by reduced iron and ROS accumulation, reduced lipid peroxidation level and restored ferroptosis-related protein expression levels.

The MCAO model is one of the models that most closely simulates human ischemic stroke (23). Animals that underwent cerebral ischemia and reperfusion displayed neurological movement disorders and pathological alterations, including brain edema and infarct area (24). In the present study, increased neurological deficit scores, brain water content and cerebra infarct areas were detected in MCAO model rats, suggesting the successful induction of ischemic stroke. However, CY alleviated MCAO-induced neurological deficit, brain edema and infarct area, indicating that CY pretreatment improved neurological function and ischemic brain injury in MCAO model rats.

Ischemic stroke has been reported to damage neurons (25). MAP-2, a protein expressed primarily in neuronal dendrites, is critical for numerous microtubule-related processes, including microtubule assembly, stabilization and cross-linking (26). MAP-2 deficiency following ischemic stroke has been documented in previous studies $(27,28)$. In the present study, decreased MAP-2 immunofluorescence activity was observed in MCAO model rats, indicating MAP-2 defect in ischemic stroke. CY inhibited MCAO-induced reductions in MAP-2, suggesting that $\mathrm{CY}$ protected rats against MCAO-induced neuron injury.

Evidence suggests that the post-ischemic inflammatory responses alter the outcome of ischemic stroke (29). NF- $\mathrm{B}$ is a pivotal regulator of inflammation-related gene transcription (30). Studies have revealed that $N F-\kappa B$ could serve as an upstream activator of the NLRP3 inflammasome, which consists of NLRP3, ASC and caspase-1, and contribute to the production of TNF- $\alpha$, IL-1 $\beta$ and IL-6 $(31,32)$. The $\mathrm{NF}-\kappa \mathrm{B} / \mathrm{NLRP} 3$ inflammasome signaling pathway has been implicated in ischemic stroke (33). Terai et al (25) examined the distribution of $\mathrm{NF}-\kappa \mathrm{B}$ in brain samples obtained from patients who died following a stroke, and discovered that the immunoreactivity of $\mathrm{NF}-\kappa \mathrm{B}$ was enhanced in glial cells of infarcted areas compared with control cases. Arctigenin, a phenylpropanoid dibenzylbutyrolactone lignan derived from Arctium lappa L, has been reported to inhibit MCAO-induced neuronal deterioration, cerebral infarct and brain water content by inhibiting NLRP3 inflammasome activation and IL-1 $\beta$ secretion (34). In the present study, NF- $\kappa \mathrm{B} / \mathrm{NLRP} 3$ inflammasome signaling expression levels were upregulated in the cortex of MCAO model rats, which was accompanied by increased levels of TNF- $\alpha$, IL- $1 \beta$ and IL-6 in the serum. Following CY administration, the aforementioned MCAO-induced effects were reversed, suggesting the involvement of NF- $\kappa \mathrm{B} / \mathrm{NLRP} 3$ inflammasome signaling in the pathology and treatment of ischemic stroke.

Ferroptosis is a newly defined cell death process that is characterized by iron-dependent accumulation of lipid peroxides to lethal levels (35). Increasing evidence has highlighted the role of ferroptosis in ischemic stroke (16). The content of free iron and MDA in brain tissues were significantly augmented, whereas levels of GPX4 and GSH were greatly decreased in MCAO model animals (36). Tau ablation protected mice against from MCAO-induced iron accumulation in the brain and inhibited ferroptosis, leading to the attenuation of the ischemia-reperfusion injury including neurological defects, infarct area, motor and cognitive dysfunctions (16). In the present study, MCAO challenge promoted cortex iron and ROS accumulation and serum lipid peroxidation levels, resulting in aberrant expression levels of ferroptosis-related proteins in the brain, whereas treatment with $\mathrm{CY}$ inhibited MCAO challenge-induced alterations. The aforementioned results suggested that ferroptosis may partially account for experimental ischemic stroke progression and CY-related treatment effects in MCAO model rats.

Collectively, the results of the present study suggested that $\mathrm{CY}$ treatment protected rats against ischemia-reperfusion injury by alleviating inflammation and ferroptosis (Fig. 10). The results indicated that $\mathrm{CY}$ may serve as a potential therapeutic agent for ischemic stroke.

\section{Acknowledgements}

Not applicable.

\section{Funding}

This work was supported by the Science and Technology Project of Jiangsu Provincial Bureau of Traditional Chinese Medicine (JD201812) and the Social Development Project of Jiangsu Science and Technology Department (BE2017769).

\section{Availability of data and materials}

The datasets used and/or analyzed during the current study are available from the corresponding author on reasonable request. 


\section{Authors' contributions}

$\mathrm{HG}, \mathrm{CB}$ and JL were involved in the design of the study. HG, LZ and PT performed the experiments. DC analyzed the data. YL interpretated the data and drafted the manuscript with the support of all authors. All authors read and approved the manuscript and agree to be accountable for all aspects of the research in ensuring that the accuracy or integrity of any part of the work are appropriately investigated and resolved.

\section{Ethics approval and consent to participate}

The study was approved by the Animal Care and Use Ethics Committee of Nanjing University of Chinese Medicine (Nanjing, China) (approval no. 201907A544).

\section{Patient consent for publication}

Not applicable.

\section{Competing interests}

The authors declare that they have no competing interests.

\section{References}

1. Cao G, Jiang N, Hu Y, Zhang Y, Wang G, Yin M, Ma X, Zhou K, Qi J, Yu B and Kou J: Ruscogenin attenuates cerebral ischemia-induced blood-brain barrier dysfunction by suppressing TXNIP/NLRP3 inflammasome activation and the MAPK pathway. Int J Mol Sci 17: 1418, 2016.

2. So PW, Ekonomou A, Galley K, Brody L, Sahuri-Arisoylu M, Rattray I, Cash D and Bell JD: Intraperitoneal delivery of acetate-encapsulated liposomal nanoparticles for neuroprotection of the penumbra in a rat model of ischemic stroke. Int J Nanomedicine 14: 1979-1991, 2019.

3. Anrather $\mathbf{J}$ and Iadecola $\mathrm{C}$ : Inflammation and stroke: An overview. Neurotherapeutics 13: 661-670, 2016.

4. Alim I, Caulfield JT, Chen Y, Swarup V, Geschwind DH, Ivanova E, Seravalli J, Ai Y, Sansing LH, Ste Marie EJ, et al: Selenium drives a transcriptional adaptive program to block ferroptosis and treat stroke. Cell 177: 1262-1279.e25, 2019.

5. Zahid A, Li B, Kombe AJK, Jin T and Tao J: Pharmacological inhibitors of the NLRP3 inflammasome. Front Immunol 10: 2538, 2019.

6. Negash AA, Olson RM, Griffin S and Gale M Jr: Modulation of calcium signaling pathway by hepatitis $\mathrm{C}$ virus core protein stimulates NLRP3 inflammasome activation. PLoS Pathog 15: e1007593, 2019.

7. Fann DY, Lim YA, Cheng YL, Lok KZ, Chunduri P, Baik SH, Drummond GR, Dheen ST, Sobey CG, Jo DG, et al: Evidence that NF- $\kappa \mathrm{B}$ and MAPK signaling promotes NLRP inflammasome activation in neurons following ischemic stroke. Mol Neurobiol 55: 1082-1096, 2018.

8. Wu H, Tang C, Tai LW, Yao W, Guo P, Hong J, Yang X, Li X, Jin Z, Ke J and Wang Y: Flurbiprofen axetil attenuates cerebral ischemia/reperfusion injury by reducing inflammation in a rat model of transient global cerebral ischemia/reperfusion. Biosci Rep 38: BSR20171562, 2018.

9. Jiang T, Wu M, Zhang Z, Yan C, Ma Z, He S, Yuan W, Pu K and Wang Q: Electroacupuncture attenuated cerebral ischemic injury and neuroinflammation through $\alpha 7 \mathrm{nAChR}$-mediated inhibition of NLRP3 inflammasome in stroke rats. Mol Med 25: 22, 2019.

10. Wang L, Liu Y, Du T, Yang H, Lei L, Guo M, Ding HF, Zhang J, Wang $\mathrm{H}$, Chen $\mathrm{X}$ and Yan C: ATF3 promotes erastin-induced ferroptosis by suppressing system Xc. Cell Death Differ 27: 662-675, 2020

11. Bai T, Lei P, Zhou H, Liang R, Zhu R, Wang W, Zhou L and Sun Y: Sigma-1 receptor protects against ferroptosis in hepatocellular carcinoma cells. J Cell Mol Med 23: 7349-7359, 2019.
12. Doll S, Proneth B, Tyurina YY, Panzilius E, Kobayashi S, Ingold I, Irmler M, Beckers J, Aichler M, Walch A, et al: ACSL4 dictates ferroptosis sensitivity by shaping cellular lipid composition. Nat Chem Biol 13: 91-98, 2017.

13. Lei G, Zhang Y, Koppula P, Liu X, Zhang J, Lin SH, Ajani JA, Xiao Q, Liao Z, Wang H and Gan B: The role of ferroptosis in ionizing radiation-induced cell death and tumor suppression. Cell Res 30: 146-162, 2020

14. Feng H, Schorpp K, Jin J, Yozwiak CE, Hoffstrom BG, Decker AM, Rajbhandari P, Stokes ME, Bender HG, Csuka JM, et al: Transferrin receptor is a specific ferroptosis marker. Cell Rep 30: 3411-3423.e7, 2020.

15. Lu J, Liu X, Tian Y, Li H, Ren Z, Liang S, Zhang G, Zhao C, Li X, Wang T, et al: Moxibustion exerts a neuroprotective effect through antiferroptosis in Parkinson's disease. Evid Based Complement Alternat Med 2019: 2735492, 2019.

16. Tuo QZ, Lei P, Jackman KA, Li XL, Xiong H, Li XL, Liuyang ZY, Roisman L, Zhang ST, Ayton S, et al: Tau-mediated iron export prevents ferroptotic damage after ischemic stroke. Mol Psychiatry 22: 1520-1530, 2017

17. Guan X, Li X, Yang X, Yan J, Shi P, Ba L, Cao Y and Wang P: The neuroprotective effects of carvacrol on ischemia/reperfusion-induced hippocampal neuronal impairment by ferroptosis mitigation. Life Sci 235: 116795, 2019.

18. Lu QY, Ma JQ, Duan YY, Sun Y, Yu S, Li B and Zhang GM: Carthamin yellow protects the heart against ischemia/reperfusion injury with reduced reactive oxygen species release and inflammatory response. J Cardiovasc Pharmacol 74: 228-234, 2019.

19. Chen B, Wang HT, Yu B, Zhang JD and Feng Y: Carthamin yellow inhibits matrix degradation and inflammation induced by LPS in the intervertebral disc via suppression of MAPK pathway activation. Exp Ther Med 14: 1614-1620, 2017.

20. Sun R, Song Y, Li S, Ma Z, Deng X, Fu Q, Qu R and Ma S: Levo-tetrahydropalmatine attenuates neuron apoptosis induced by cerebral ischemia-reperfusion injury: Involvement of c-Abl activation. J Mol Neurosci 65: 391-399, 2018.

21. Yang Y, Hu Z, Du X, Davies H, Huo X and Fang M: miR-16 and fluoxetine both reverse autophagic and apoptotic change in chronic unpredictable mild stress model rats. Front Neurosci 11: $428,2017$.

22. Chen S, Dong Z, Cheng M, Zhao Y, Wang M, Sai N, Wang X, Liu H, Huang G and Zhang X: Homocysteine exaggerates microglia activation and neuroinflammation through microglia localized STAT3 overactivation following ischemic stroke. J Neuroinflammation 14: 187, 2017.

23. Fluri F, Schuhmann MK and Kleinschnitz C: Animal models of ischemic stroke and their application in clinical research. Drug Des Devel Ther 9: 3445-3454, 2015.

24. Guo P, Jin Z, Wu H, Li X, Ke J, Zhang Z and Zhao Q: Effects of irisin on the dysfunction of blood-brain barrier in rats after focal cerebral ischemia/reperfusion. Brain Behav 9: e01425, 2019.

25. Terai K, Matsuo A, McGeer EG and McGeer PL: Enhancement of immunoreactivity for NF-kappa B in human cerebral infarctions. Brain Res 739: 343-349, 1996.

26. Gumy LF, Katrukha EA, Grigoriev I, Jaarsma D, Kapitein LC, Akhmanova A and Hoogenraad CC: MAP2 defines a pre-axonal filtering zone to regulate KIF1-versus KIF5-dependent cargo transport in sensory neurons. Neuron 94: 347-362.e7, 2017.

27. He F, Dai R, Zhou X, Li X, Song X, Yan H, Meng Q, Yang C and Lin Q: Protective effect of 4-Methoxy benzyl alcohol on the neurovascular unit after cerebral ischemia reperfusion injury. Biomed Pharmacother 118: 109260, 2019.

28. Xu SY, Hu YF, Li WP, Wu YM, Ji Z, Wang SN, Li K and Pan SY Intermittent hypothermia is neuroprotective in an in vitro model of ischemic stroke. Int J Biol Sci 10: 873-881, 2014.

29. Lambertsen KL, Finsen B and Clausen BH: Post-stroke inflammation-target or tool for therapy? Acta Neuropathol 137: 693-714, 2019.

30. Hoesel B and Schmid JA: The complexity of NF- $\kappa B$ signaling in inflammation and cancer. Mol Cancer 12: 86, 2013.

31. Yu X, Lan P, Hou X, Han Q, Lu N, Li T, Jiao C, Zhang J, Zhang C and Tian Z: HBV inhibits LPS-induced NLRP3 inflammasome activation and IL-1 $\beta$ production via suppressing the $N F-\kappa B$ pathway and ROS production. J Hepatol 66: 693-702, 2017.

32. Zeng J, Chen Y, Ding R, Feng L, Fu Z, Yang S, Deng X, Xie Z and Zheng S: Isoliquiritigenin alleviates early brain injury after experimental intracerebral hemorrhage via suppressing ROSand/or NF- $\kappa \mathrm{B}$-mediated NLRP3 inflammasome activation by promoting Nrf2 antioxidant pathway. J Neuroinflammation 14: 119,2017 
33. Ye Y, Jin T, Zhang X, Zeng Z, Ye B, Wang J, Zhong Y, Xiong X and $\mathrm{Gu}$ L: Meisoindigo protects against focal cerebral ischemia-reperfusion injury by inhibiting NLRP3 inflammasome activation and regulating microglia/macrophage polarization via TLR4/NF- $\mathrm{BB}$ signaling pathway. Front Cell Neurosci 13: 553, 2019.

34. Zhang S, Jiang L, Che F, Lu Y, Xie Z and Wang H: Arctigenin attenuates ischemic stroke via SIRT1-dependent inhibition of NLRP3 inflammasome. Biochem Biophys Res Commun 493: 821-826, 2017.
35. Weiland A, Wang Y, Wu W, Lan X, Han X, Li Q and Wang J: Ferroptosis and its role in diverse brain diseases. Mol Neurobiol 56: 4880-4893, 2019.

36. Lu J, Xu F and Lu H: LncRNA PVT1 regulates ferroptosis through miR-214-mediated TFR1 and p53. Life Sci 260: 118305, 2020.

cc) (i) $\ominus$ This work is licensed under a Creative Commons Attribution-NonCommercial-NoDerivatives 4.0 International (CC BY-NC-ND 4.0) License. 